\title{
Weighted Local Time for Fractional Brownian Motion and Applications to Finance
}

\author{
Yaozhong $\mathrm{Hu}^{*} \quad$ Bernt Øksendal Donna Mary Salopek ${ }^{\dagger}$ \\ University of Kansas, University of Oslo, York University and UNSW
}

\begin{abstract}
A Meyer-Tanaka formula involving weighted local time is derived for fractional Brownian motion and geometric fractional Brownian motion. The formula is applied to the study of the stop-loss-start-gain (SLSG) portfolio in a fractional Black-Scholes market. As a consequence, we obtain a fractional version of the Carr-Jarrow decomposition of the European call and put option prices into their intrinsic and time values.
\end{abstract}

\section{Introduction}

Fractional Brownian motion $B^{(H)}=\left\{B_{t}^{(H)}, t \geq 0\right\}$ with Hurst parameter $H \in(0,1)$, is a centered Gaussian process with $B_{0}^{(H)}=0$ and

$$
\operatorname{Cov}\left(B_{t}^{(H)}, B_{s}^{(H)}\right)=\frac{1}{2} \operatorname{Var}\left(B_{1}^{(H)}\right)\left[|t|^{2 H}+|s|^{2 H}-|t-s|^{2 H}\right]
$$

for all $t, s \geq 0$. Since the paper of Mandelbrot and van Ness (1968), finding a stochastic calculus applicable to fractional Brownian motion has become an very active research area. For instance, a number of Itô formula extensions have been suggested by Alòs, Mazet and Nualart [1], Dai and Heyde [7], Duncan Hu and Pasik-Duncan [8], Hu and Øksendal [13] , Lin [18] and Norvaiša [20], and each of these papers offers a different approach to obtaining a chain rule for fractional Brownian motion.

In this paper, we provide a Meyer-Tanaka formula for both fractional Brownian motion and geometric fractional Brownian motion. In Section 2, we review the definitions and

AMS 1991 subject classifications: Primary 60H05, 60H10;

Key words and phrases: Fractional Brownian motions; Local time; Chaos expansion; Asymptotic behavior.

*Partially supported by the National Science Foundation under Grant No. EPS-9874732 and matching support from the State of Kansas.

${ }_{\dagger}^{\dagger}$ Partially supported by NSERC Canada grant number 203232-98 York University. 
certain properties of weighted local times (the weighted local time is a more convenient way to describe the generalized Itô formula that we discuss in this paper). In Section 3, we introduce the notions of generalized stochastic integrals, and in Section 4, we obtain a generalized version of the Meyer-Tanaka formula for the reflection of fractional Brownian motion. In Section 5, we prove a Meyer-Tanaka formula for geometric fractional Brownian motion and state certain properties of the weighted local times of geometric fractional Brownian motion. In the final section, this generalized Itô formula will be applied to the SLSG portfolio in a fractional Black and Scholes market.

While revising this paper, the authors became aware of a recent paper by Coutin, Nualart and Tudor [6], where a similar Meyer-Tanaka formula is obtained for fractional Brownian motion with Hurst parameter $H>1 / 3$. Their local time $L_{t}^{a}$ is the same as our weighted local time $\mathcal{L}_{t}^{\left(B^{H}\right)}$. The methods used are different. Moreover, our approach works for any $H \in(0,1)$ and it can be used to find the local time of functions of fractional Brownian motion.

\section{Weighted Local Times}

In this and the remaining sections we fix $H \in(0,1)$. Let $B^{(H)}=\left\{B_{t}^{(H)}, t \geq 0\right\}$ be a 1dimensional fractional Brownian motion with Hurst parameter $H$. The usual local time $\ell_{t}^{\left(B^{(H)}\right)}(x)$ of $B^{(H)}$ at $x \in \mathbb{R}$, is defined by

$$
\ell_{t}^{\left(B^{(H)}\right)}(x)=\int_{0}^{t} \delta\left(B_{s}^{(H)}-x\right) d s=\lim _{\varepsilon \rightarrow 0} \frac{1}{2 \varepsilon}\left|\left\{s \in[0, t] ;\left|B_{s}^{(H)}-x\right|<\varepsilon\right\}\right|,
$$

where $\delta$ is the Dirac $\delta$ function and $|A|$ denotes the Lebesgue measure of a Borel set $A$. It is proved in Hu and $\varnothing \mathrm{ksendal}$ [14] that for every $t$ and $x$, the local time $\ell_{t}^{\left(B^{(H)}\right)}(x)$ is an element in $L^{2}(\Omega, \mathcal{F}, P)$.

Now we introduce the weighted local time as

$$
\mathcal{L}_{t}^{\left(B^{(H)}\right)}(x)=\int_{0}^{t} \delta\left(B_{s}^{(H)}-x\right) s^{2 H-1} d s .
$$

For $H=1 / 2$, the usual local time is the same as the weighted local time. The weighted local time $\mathcal{L}_{t}^{\left(B^{(H)}\right)}(x)$ is a jointly continuous function of $t$ and $x$ for almost all $\omega \in \Omega$ (see Berman [3]). Following the proof of [3], we also see easily that the local time $\ell_{t}^{\left(B^{(H)}\right)}(x)$ is a jointly continuous function of $t$ and $x$ for almost all $\omega \in \Omega$. With this in mind, we have the following proposition.

\section{Proposition 2.1}

$$
\begin{aligned}
& \mathbb{E}\left[\ell_{t}^{\left(B^{(H)}\right)}(x)\right]=(2 \pi)^{-1 / 2} \int_{0}^{t} r^{-H} \exp \left(-\frac{1}{2} r^{-2 H} x^{2}\right) d r \\
& \mathbb{E}\left[\mathcal{L}_{t}^{\left(B^{(H)}\right)}(x)\right]=(2 \pi)^{-1 / 2} \int_{0}^{t} r^{H-1} \exp \left(-\frac{1}{2} r^{-2 H} x^{2}\right) d r .
\end{aligned}
$$


Proof. The proof of representation (2.2) can be found in $\mathrm{Hu}$ and Øksendal ([14], Corollary $4.10 . \mathrm{b})$. To prove (2.3), use integration by parts to write

$$
\begin{aligned}
\mathcal{L}_{t}^{\left(B^{(H)}\right)}(x) & =\int_{0}^{t} \delta\left(B_{s}^{(H)}-x\right) s^{2 H-1} d s \\
& =\left.\ell_{s}^{\left(B^{(H)}\right)}(x) s^{2 H-1}\right|_{0} ^{t}-(2 H-1) \int_{0}^{t} s^{2 H-2} \ell_{s}^{\left(B^{(H)}\right)}(x) d s \\
& =\ell_{t}^{\left(B^{(H)}\right)}(x) t^{2 H-1}-(2 H-1) \int_{0}^{t} s^{2 H-2} \ell_{s}^{\left(B^{(H)}\right)}(x) d s .
\end{aligned}
$$

By (2.2), we have

$$
\begin{aligned}
\mathbb{E}\left\{\int_{0}^{t} s^{2 H-2} \ell_{s}^{\left(B^{(H)}\right)}(x) d s\right\}= & \int_{0}^{t} s^{2 H-2}\left(\frac{1}{\sqrt{2 \pi}} \int_{0}^{s} r^{-H} \exp \left(-\frac{1}{2} r^{-2 H} x^{2}\right) d r\right) d s \\
= & \frac{1}{\sqrt{2 \pi}} \int_{0}^{t}\left(\int_{r}^{t} s^{2 H-2} d s\right) r^{-H} \exp \left(-\frac{1}{2} r^{-2 H} x^{2}\right) d r \\
= & \frac{1}{\sqrt{2 \pi}} \int_{0}^{t} \frac{1}{2 H-1}\left(t^{2 H-1}-r^{2 H-1}\right) r^{-H} \exp \left(-\frac{1}{2} r^{-2 H} x^{2}\right) d r \\
= & \frac{t^{2 H-1}}{2 H-1}\left(\mathbb{E}\left[\ell_{t}^{\left(B^{(H)}\right)}(x)\right]\right) \\
& -\frac{1}{(2 H-1) \sqrt{2 \pi}} \int_{0}^{t} r^{H-1} \exp \left(-\frac{1}{2} r^{-2 H} x^{2}\right) d r
\end{aligned}
$$

Combining (2.4) and (2.5), we obtain (2.3).

The exact second moment is harder to compute. However, we are able to obtain an upper bound for the second moment.

Proposition 2.2 The weighted local time $\mathcal{L}_{T}^{\left(B^{(H)}\right)}(x)=\int_{0}^{T} \delta\left(B^{(H)}(t)-x\right) t^{2 H-1} d t$ is square integrable and for any $x \in \mathbb{R}$, we have

$$
\mathbb{E}\left[\mathcal{L}_{T}^{\left(B^{(H)}\right)}(x)\right]^{2} \leq \frac{\Gamma(H) \Gamma(1-H) T^{2 H}}{2 H \pi \sqrt{k}},
$$

where $k$ is a constant depending on $H$ and is defined by (2.6) below.

Proof. Using the representation (2.1), we follow the proof of Theorem 5.3 of $\mathrm{Hu}$ and Øksendal [14] and get

$$
\begin{aligned}
\mathbb{E}\left\{\left[\mathcal{L}_{T}^{\left(B^{(H)}\right)}(x)\right]^{2}\right\} & =\mathbb{E}\left[\int_{0}^{T} \delta\left(B^{(H)}(t)-x\right) t^{2 H-1} d t\right]^{2} \\
& =\frac{1}{(2 \pi)^{2}} \int_{[0, T]^{2}} \int_{\mathbb{R}^{2}} e^{-(i \xi x+i \eta x)} \mathbb{E} e^{i \xi B^{(H)}(t)+i \eta B^{(H)}(s)} d \xi d \eta(s t)^{2 H-1} d s d t
\end{aligned}
$$




$$
\begin{aligned}
& \leq \frac{1}{(2 \pi)^{2}} \int_{[0, T]^{2}} \int_{\mathbb{R}^{2}} e^{-\frac{1}{2} \operatorname{Var}\left(\xi B^{(H)}(t)-\eta B^{(H)}(s)\right)} d \xi d \eta(s t)^{2 H-1} d s d t \\
& \leq \frac{2}{(2 \pi)^{2}} \int_{0 \leq s<t \leq T} \int_{\mathbb{R}^{2}} e^{-\frac{1}{2} \operatorname{Var}\left(\xi B^{(H)}(t)-\eta B^{(H)}(s)\right)} d \xi d \eta(s t)^{2 H-1} d s d t .
\end{aligned}
$$

Using the well-known nondeterminism property of fractional Brownian motions (see Berman [3], $\mathrm{Hu}$ and Øksendal [14], $\mathrm{Hu}$ [11], and the reference therein), we find that, when $0 \leq s<$ $t \leq T$, there is a positive constant $k>0$ such that

$$
\begin{aligned}
\operatorname{Var}\left(\xi B^{(H)}(t)-\eta B^{(H)}(s)\right) & =\operatorname{Var}\left(\xi\left(B^{(H)}(t)-B^{(H)}(s)\right)+(\xi-\eta) B^{(H)}(s)\right) \\
& \geq k\left[\xi^{2}|t-s|^{2 H}+(\eta-\xi)^{2} s^{2 H}\right]
\end{aligned}
$$

Therefore, we have

$$
\begin{aligned}
\mathbb{E}\left\{\left[\mathcal{L}_{T}^{\left(B^{(H)}\right)}(x)\right]^{2}\right\} & \leq \frac{2}{(2 \pi)^{2}} \int_{0 \leq s<t \leq T} \int_{\mathbb{R}^{2}} e^{-\frac{k}{2}\left(\xi^{2}|t-s|^{2 H}+(\eta-\xi)^{2} s^{2 H}\right)}(s t)^{2 H-1} d \xi d \eta d s d t \\
& =\frac{2}{2 \pi \sqrt{k}} \int_{0 \leq s<t \leq T} \frac{(s t)^{2 H-1}}{s^{H}(t-s)^{H}} d s d t \\
& =\frac{2}{2 \pi \sqrt{k}} \int_{0}^{T}\left(\int_{0}^{t} \frac{s^{H-1} t^{2 H-1}}{(t-s)^{H}} d s\right) d t \\
& =\frac{2 \Gamma(H) \Gamma(1-H)}{2 \pi \sqrt{k}} \int_{0}^{T} t^{2 H-1} d t \\
& =\frac{\Gamma(H) \Gamma(1-H)}{2 H \pi \sqrt{k}} T^{2 H} .
\end{aligned}
$$

This proves the proposition.

\section{Generalized Stochastic Integrals}

In this section, we briefly recall the definition and the properties of the Wick-Itô integral

$$
\int_{\mathbb{R}} Y(s) d B_{s}^{(H)}
$$

of a process $Y(s)$. This integral was first developed for the case $\frac{1}{2}<H<1$ by Duncan, Hu, and Pasik-Duncan [8] and subsequently, in a white noise setting by $\mathrm{Hu}$ and Øksendal [13]. Here we present the extension to the general case $0<H<1$, which was recently obtained by Elliott and van der Hoek [10]. 
Let $(\mathcal{S})^{*}$ be the Hida space of stochastic distributions and let $\diamond$ denote the Wick product on $(\mathcal{S})^{*}$ (see e.g. Holden, Øksendal, Ubøe, and Zhang [12] for definitions and properties). Let

$$
W_{t}^{(H)}=\frac{d}{d t} B_{t}^{(H)} \quad\left(\text { derivative in }(\mathcal{S})^{*}\right)
$$

be the fractional white noise.

If $Y: \mathbb{R} \rightarrow(\mathcal{S})^{*}$ we define the (generalized) Wick-Itô integral of $Y$ with respect to $B_{t}^{(H)}$ by

$$
\int_{\mathbb{R}} Y(t) d B_{t}^{(H)}=\int_{\mathbb{R}} Y(t) \diamond W_{t}^{(H)} d t
$$

whenever the last integral exists as an integral in $(\mathcal{S})^{*}$. Then it is not hard to show that this integral is an extension of the integral introduced by Duncan et al [8] (also see Hu and Øksendal [13]). In particular, we have:

Lemma 3.1 (The fractional Itô formula) Let $f: \mathbb{R} \times \mathbb{R} \rightarrow \mathbb{R}$ be twice continuously differentiable and such that, with $f^{\prime \prime}(s, x)=\frac{\partial^{2} f}{\partial x^{2}}(s, x)$,

$$
\int_{0}^{T} s^{2 H-1}\left|f^{\prime \prime}\left(s, B_{s}^{(H)}\right)\right| d s<\infty \quad \text { a.s.. }
$$

Then, for all $t \in[0, T]$, we have with $f^{\prime}(s, x)=\frac{\partial f}{\partial x}(s, x)$,

$$
\begin{aligned}
& f\left(t, B_{t}^{(H)}\right)=f(0,0)+\int_{0}^{t} \frac{\partial f}{\partial s}\left(s, B_{s}^{(H)}\right) d s \\
& \quad+\int_{0}^{t} f^{\prime}\left(s, B_{s}^{(H)}\right) d B_{s}^{(H)}+H \int_{0}^{t} s^{2 H-1} f^{\prime \prime}\left(s, B_{s}^{(H)}\right) d s \quad \text { a.s. }
\end{aligned}
$$

This result was first proved by Duncan et al [8] for the case $\frac{1}{2}<H<1$, and the above general case has been obtained independently by Biagini et al [4], Bender [2] and J. van der Hoek [23].

\section{A Generalized Itô Formula}

Let $f: \mathbb{R} \rightarrow \mathbb{R}$ be a convex function. Then it is well-known that its left derivative $D^{-} f(x)$ exists and is finite for every $x \in \mathbb{R}$. Define the second derivative measure of $f$ by $\nu_{f}(d x)$ by

$$
\nu_{f}([a, b)):=D^{-} f(b)-D^{-} f(a) ; \quad-\infty<a<b<\infty .
$$

Theorem 4.1 Let $f$ be a convex function of polynomial growth. Then

$$
f\left(B_{t}^{(H)}\right)=f(0)+\int_{0}^{t} D^{-} f\left(B_{s}^{(H)}\right) d B_{s}^{(H)}+H \int_{\mathbb{R}} \mathcal{L}_{t}^{\left(B^{(H)}\right)}(x) \nu_{f}(d x) .
$$


Proof. Define a function $\rho$ by

$$
\rho(x):=c \exp \left\{\frac{1}{(x-1)^{2}-1}\right\}
$$

for $x \in(0,2)$, and $\rho(x)=0$ elsewhere, where $c$ is a normalizing constant such that $\int_{\mathbb{R}} \rho(x) d x=1$. Let

$$
\rho_{n}(x):=n \rho(n x)
$$

If

$$
f_{n}(x)=\int_{\mathbb{R}} \rho_{n}(x-y) f(y) d y, \quad n \geq 1
$$

then it is well-known that

$$
\lim _{n \rightarrow \infty} f_{n}(x)=f(x), \quad \text { and } \quad \lim _{n \rightarrow \infty} f_{n}^{\prime}(x)=D^{-} f(x),
$$

for every $x \in \mathbb{R}$. Moreover, if $g$ is of class $C^{1}$ and has compact support, then

$$
\lim _{n \rightarrow \infty} \int_{\mathbb{R}} g(x) f_{n}^{\prime \prime}(x) d x=\int_{\mathbb{R}} g(x) \nu_{f}(d x) .
$$

Since $f_{n}$ is $C^{2}$, we have by Lemma 3.1

$$
f_{n}\left(B_{t}^{(H)}\right)=f_{n}(0)+\int_{0}^{t} f_{n}^{\prime}\left(B_{s}^{(H)}\right) d B_{s}^{(H)}+H \int_{0}^{t} s^{2 H-1} f_{n}^{\prime \prime}\left(B_{s}^{(H)}\right) d s .
$$

If $n \rightarrow \infty$, then it is easy to see that $f_{n}\left(B_{t}^{(H)}\right)$ converges to $f\left(B_{t}^{(H)}\right)$ almost surely, and $f_{n}(0)$ converges to $f(0)$.

Next we consider the limit of the last term. We have

$$
\begin{aligned}
H \int_{0}^{t} s^{2 H-1} f_{n}^{\prime \prime}\left(B_{s}^{(H)}\right) d s & =H \int_{0}^{t} s^{2 H-1} \int_{\mathbb{R}} f_{n}^{\prime \prime}(x) \delta\left(B_{s}^{(H)}-x\right) d x d s \\
& =H \int_{\mathbb{R}} f_{n}^{\prime \prime}(x) \int_{0}^{t} s^{2 H-1} \delta\left(B_{s}^{(H)}-x\right) d s d x \\
& =H \int_{\mathbb{R}} f_{n}^{\prime \prime}(x) \mathcal{L}_{t}^{\left(B^{(H)}\right)}(x) d x \\
& \rightarrow H \int_{\mathbb{R}} \mathcal{L}_{t}^{\left(B^{(H)}\right)}(x) \nu_{f}(d x) .
\end{aligned}
$$

Finally we see that

$$
\int_{0}^{t} f_{n}^{\prime}\left(B_{s}^{(H)}\right) \diamond W_{s}^{(H)} d s \rightarrow \int_{0}^{t} D^{-} f\left(B_{s}^{(H)}\right) \diamond W_{s}^{(H)} d s \quad \text { in }(\mathcal{S})^{*}
$$

and also almost surely, by the convergence of the other terms in (4.2). So, the result follows.

The function $f$ defined by $f(x)=|x-z|$ for $x \in \mathbb{R}$ is convex, $D^{-} f(x)=\operatorname{sign}(x-z)$, and $\nu_{f}(d x)=2 \delta_{z}(d x)$. Thus we obtain the following representation for the reflection of fractional Brownian motion. 


\section{Corollary 4.2 (A Meyer-Tanaka formula for fractional Brownian motion)}

For any $z \in \mathbb{R}$,

$$
\left|B_{t}^{(H)}-z\right|=|z|+\int_{0}^{t} \operatorname{sign}\left(B_{s}^{(H)}-z\right) d B_{s}^{(H)}+2 H \mathcal{L}_{t}^{\left(B^{(H)}\right)}(z) .
$$

As stated in the introduction, Coutin, Nualart, and Tudor [6] have also obtained a MeyerTanaka formula for fractional Brownian motion.

\section{A Meyer-Tanaka Formula For Geometric Fractional Brownian Motions}

Let $X_{t}$ be a geometric fractional Brownian motion, defined by

$$
d X_{t}=\mu X_{t} d t+\sigma X_{t} d B_{t}^{(H)}, \quad t \geq 0 ; \quad X_{0}=x>0, \quad \text { aconstant } .
$$

Using the Wick calculus (see Hu and Øksendal ([13], Example 3.14) and Elliott and van der Hoek ([10], Section 6)), the solution of this equation is found to be

$$
X_{t}=x \exp \left(\sigma B_{t}^{(H)}+\mu t-\frac{1}{2} \sigma^{2} t^{2 H}\right) .
$$

For this process, we define the local time $\ell_{t}^{(X)}(z)$ of $X$ at the point $z>0$ by

$$
\ell_{t}^{(X)}(z)=\int_{0}^{t} \delta\left(X_{s}-z\right) d s=\lim _{\varepsilon \rightarrow 0} \frac{1}{2 \varepsilon}\left|\left\{s \in[0, t] ;\left|X_{s}-z\right|<\varepsilon\right\}\right|,
$$

and the weighted local time $\mathcal{L}_{t}^{(X)}(z)$ of $X$ at $z$ by

$$
\mathcal{L}_{t}^{(X)}(z)=\int_{0}^{t} \delta\left(X_{s}-z\right) s^{2 H-1} d s .
$$

Since $X_{t}$ is a functional of $B_{t}^{(H)}$, we expect that the local time of $X$ will also be related the local time of $B^{(H)}$. The approach that we are going to develop is applicable more generally to any process of the form $Y_{t}=f\left(t, B^{(H)}(t)\right)$.

Let $f(t, x)$ be a continuous function of $t$ and $x$ such that as a function of $x$ it is invertible with continuously differentiable inverse function $f^{-1}(t, x)$. Then for any smooth function $\phi$ of compact support we have, fixing $t$ and writing $f(y)=f(t, y)$,

$$
\int_{\mathbb{R}} \delta(f(y)-z) \phi(z) d z=\phi(f(y)) .
$$

On the other hand, if we make the substitution $u=f^{-1}(z)$, we get

$$
\begin{aligned}
\int_{\mathbb{R}} \delta\left(y-f^{-1}(z)\right) \frac{d}{d z}\left[f^{-1}(z)\right] \phi(z) d z & =\int_{\mathbb{R}} \delta(y-u) \phi(f(u)) d u \\
& =\phi(f(y)) .
\end{aligned}
$$


Thus we have

$$
\delta(f(y)-z)=\delta\left(y-f^{-1}(z)\right) \frac{d}{d z}\left[f^{-1}(z)\right]
$$

(in the distribution sense). Now we consider the local time of $Y_{t}=f\left(t, B^{(H)}(t)\right)$ with

$$
f(t, y)=x \exp \left(\sigma y+\mu t-\frac{1}{2} \sigma^{2} t^{2 H}\right) .
$$

Denote the inverse of $y \rightarrow f(t, y)$ by $h(t, y)$. Then

$$
h(t, z)=\frac{1}{\sigma}\left[\log \frac{z}{x}-\mu t+\frac{\sigma^{2}}{2} t^{2 H-1}\right] .
$$

The derivative of $h(t, z)$ with respect to $z$ is

$$
\frac{d}{d z} h(t, z)=\frac{d}{d z}\left[f^{-1}(t, z)\right]=\frac{1}{\sigma z} .
$$

Thus

$$
\begin{aligned}
\mathcal{L}_{t}^{(X)}(z) & =\int_{0}^{t} \delta\left(f\left(s, B^{(H)}(s)\right)-z\right) s^{2 H-1} d s \\
& =\int_{0}^{t} \delta\left(B^{(H)}(s)-h(s, z)\right) \frac{1}{\sigma z} s^{2 H-1} d s \\
& =\frac{1}{2 \sigma z \pi} \int_{0}^{t} \int_{\mathbb{R}} e^{i \xi\left(B^{(H)}(s)-h(s, z)\right)} d \xi s^{2 H-1} d s .
\end{aligned}
$$

Now it is easy to see from our previous calculations of the first and second moments of fractional Brownian motion (see Propositions 2.1 and 2.2), that we have the following result.

\section{Proposition 5.1}

$$
\mathbb{E}\left[\mathcal{L}_{t}^{(X)}(z)\right]=\frac{1}{\sqrt{2 \pi} \sigma z} \int_{0}^{t} s^{H-1} e^{-\frac{h(s, z)^{2}}{2 s^{2 H}}} d s,
$$

where $h(s, z)$ is defined by (5.6), and

$$
\mathbb{E}\left[\mathcal{L}_{t}^{(X)}(z)\right]^{2} \leq \frac{\Gamma(H) \Gamma(1-H) t^{2 H}}{2 H \pi \sqrt{k} \sigma^{2} z^{2}} .
$$

Remark: Similarly, we can obtain

$$
\mathbb{E}\left[\ell_{t}^{(X)}(z)\right]=\frac{1}{\sqrt{2 \pi} \sigma z} \int_{0}^{t} s^{-H} e^{-\frac{h(s, z)^{2}}{2 s^{2 H}}} d s .
$$


It is interesting to note that when $f$ is independent of $t$, i.e. $Y_{t}=f\left(B^{(H)}(t)\right)$, then the local time of $Y_{t}$ is given by

$$
\begin{aligned}
\mathcal{L}_{t}^{(Y)}(f(y)) & =\int_{0}^{t} \delta\left(f\left(B^{(H)}(s)\right)-f(y)\right) s^{2 H-1} d s \\
& =\int_{0}^{t} \delta\left(B^{(H)}(s)-y\right) s^{2 H-1} \frac{1}{f^{\prime}(y)} d s \\
& =\frac{1}{f^{\prime}(y)} \int_{0}^{t} \delta\left(B^{(H)}(s)-y\right) s^{2 H-1} d s \\
& =\frac{1}{f^{\prime}(y)} \mathcal{L}_{t}^{\left(B^{(H)}\right)}(y) .
\end{aligned}
$$

This means that we have

$$
\mathcal{L}_{t}^{\left(f\left(B^{(H)}\right)\right)}(f(y))=\frac{1}{f^{\prime}(y)} \mathcal{L}_{t}^{\left(B^{(H)}\right)}(y)
$$

It is interesting to compare this formula to those in the semimartingale case (see Revuz and Yor ([21], p.234)).

The above calculations also prove the following theorem.

Theorem 5.2 The weighted local time $\mathcal{L}_{t}^{(X)}(z)$ exists as an element of $L^{2}(P)$ and it is a positive, jointly continuous function of $t>0$ and $z>0$.

Furthermore, we have the following theorem and its corollaries, the proofs of which are given in the Appendix.

Theorem 5.3 Let $f: \mathbb{R}^{+} \rightarrow \mathbb{R}$ be a convex function having polynomial growth. Then

$$
f\left(X_{t}\right)=f\left(X_{0}\right)+\int_{0}^{t} D^{-} f\left(X_{s}\right) d X_{s}+\sigma^{2} H \int_{\mathbb{R}} x^{2} \mathcal{L}_{t}^{(X)}(x) d \nu_{f}(x),
$$

where, as before, $D^{-} f$ denotes the left derivative of $f$ and $\nu_{f}$ denotes the second derivative measure of $f$.

\section{Corollary 5.4 (A Meyer-Tanaka formula for geometric fractional Brownian mo-} tion)

For any $z>0$ we have

$$
\left|X_{t}-z\right|=\left|X_{0}-z\right|+\int_{0}^{t} \operatorname{sign}\left(X_{s}-z\right) d X_{s}+2 \sigma^{2} H z^{2} \mathcal{L}_{t}^{(X)}(z)
$$

Corollary 5.5 For any $z>0$ we have

$$
\left(X_{t}-z\right)^{+}=\left(X_{0}-z\right)^{+}+\int_{0}^{t} \chi_{[z, \infty)}\left(X_{s}\right) d X_{s}+\sigma^{2} H z^{2} \mathcal{L}_{t}^{(X)}(z) .
$$




\section{Application to Finance}

We now apply Corollary 5.5 to prove that the fractional analogue of the stop-loss-startgain (SLSG) portfolio is not self-financing in a fractional Black-Scholes market. Our result includes the classical Black-Scholes market $(H=1 / 2)$ which was first proved by Carr and Jarrow [5]. For further information on this portfolio from a $p$-variation approach, see Salopek $[22]$ and the references therein.

The fractional Black-Scholes market consists of a bank account or a bond, and a stock. The price process $A_{t}$ of the bond, at time $t$ is given by

$$
d A_{t}=\rho A_{t} d t ; \quad A_{0}=1, \quad 0 \leq t \leq T,
$$

where $\rho \geq 0, T>0$ are constants. The price process $X_{t}$ of the stock is given by a geometric fractional Brownian motion

$$
d X_{t}=\mu X_{t} d t+\sigma X_{t} d B_{t}^{(H)}, \quad X_{0}=x>0,
$$

where $\mu$ and $\sigma \neq 0$ are constants. The solution of (6.2) is given by Eq.(5.2). It is known that this market has no arbitrage. That is, this financial market is complete and there exists an explicit fractional Black-Scholes formula for the price of European call option (see $\mathrm{Hu}$ and Øksendal ([13], Section 5) and Elliott and van der Hoek [10] for more details). Related issues about this market are discussed in Elliott and van der Hoek ([9],[10]), Hu and Øksendal [13], and $\mathrm{Hu}$, Oksendal and Sulem ([15], [16], [17]).

By normalizing the market (i.e. by considering all prices in units of $A_{t}$ ) we may assume without loss of generality, that

$$
\rho=0 \text {, i.e. } A_{t}=1 \text { for all } t \text {. }
$$

A European call option in this market gives the payoff

$$
F(\omega)=\left(X_{T}(\omega)-q\right)^{+}
$$

at time $T$, for some given constant $q>0$ (the exercise price of the option).

The SLSG portfolio $\theta_{t}=\left(\alpha_{t}, \beta_{t}\right)$ for this payoff is defined by

$$
\begin{gathered}
\alpha_{t}=-q \chi_{[q, \infty)}\left(X_{t}\right) \quad(\text { number of bonds or monetary units held at time } \mathrm{t}) \\
\beta_{t}=\chi_{[q, \infty)}\left(X_{t}\right) \quad(\text { number of stocks held at time } \mathrm{t}) .
\end{gathered}
$$

We may describe this as follows: Assume that we start with initial fortune 0 and $X_{0}<q$. As long as $X_{t}<q$, we do nothing. However, as soon as $X_{t} \geq q$, we immediately borrow the amount $q$ from the bank to buy one stock at the price $q$. We keep this stock until its price drops below $q$. Then we sell it immediately and pay $q$ back to the bank. The value $V_{t}^{\theta}$ at time $t$ of this portfolio $\theta$ is given by

$$
\begin{aligned}
V_{t}^{\theta} & =\alpha_{t} A_{t}+\beta_{t} X_{t} \\
& =-q \chi_{[q, \infty)}\left(X_{t}\right)+\chi_{[q, \infty)}\left(X_{t}\right) X_{t} \\
& =\left(X_{t}-q\right)^{+} .
\end{aligned}
$$


Thus this portfolio replicates the payoff $\left(X_{t}-q\right)^{+}$at all times $t \leq T$ with initial fortune 0 when $X_{0}<q$. Recall that $\theta$ is self-financing if and only if

$$
d V_{t}^{\theta}=\alpha_{t} d A_{t}+\beta_{t} d X_{t}
$$

That is, with $\rho=0$, we have

$$
d V_{t}^{\theta}=\chi_{[q, \infty)}\left(X_{t}\right) d X_{t}
$$

By (6.7), this implies that

$$
\left(X_{t}-q\right)^{+}=\left(X_{0}-q\right)^{+}+\int_{0}^{t} \chi_{[q, \infty)}\left(X_{s}\right) d X_{s} .
$$

However, Corollary 5.5 states that

$$
\left(X_{t}-q\right)^{+}=\left(X_{0}-q\right)^{+}+\int_{0}^{t} \chi_{[q, \infty)}\left(X_{s}\right) d X_{s}+\sigma^{2} H q^{2} \mathcal{L}_{t}^{(X)}(q) .
$$

Since $\mathcal{L}_{t}^{(X)}(q)>0$ for all $t>0$, we see that (6.11) contradicts (6.10). Hence $\theta$ is not selffinancing. This agrees with the fractional Black-Scholes formula given in $\mathrm{Hu}$ and Øksendal ([13], Corollary 5.3) and Elliott and van der Hoek ([10], Section 6).

Thus in this model, we are able to extend the SLSG portfolio from the geometric Brownian motion case of Carr and Jarrow [5] to a geometric fractional Brownian motion. The fact that $\theta$ is not self-financing implies that our fractional Black-Scholes model does not allow for arbitrage opportunities, and complements the results of $\mathrm{Hu}$ and Øksendal [13] and Elliott and van der Hoek ([9], [10]). Further, we obtain new expressions for the value of European call and put options in our fractional Brownian motion model. Like Carr and Jarrow [5], our expressions decompose option prices into their intrinsic and time values. That is, we have

Proposition 6.1 (An alternative call valuation formula): Consider a European call option with market value $C(0)$, maturity date $T$, and exercise price $q$. Assume the fractional Brownian motion model for $H \in(0,1)$. Then

$$
C(0)=\left(X_{0}-q\right)^{+}+\sigma^{2} H q^{2} \widetilde{E}\left[\mathcal{L}_{T}^{(X)}(q)\right]
$$

where $\widetilde{E}$ denotes the expectation under the risk-free measure $\widetilde{P}$ defined in the fractional Girsanov theorem given by Elliott and van der Hoek ([10], Section 4).

Proof. By Corollary 5.6 in Hu and Øksendal [13], Elliott and van der Hoek ([10], Section $6)$, we know that

$$
C(0)=\widetilde{E}\left[\left(X_{T}-q\right)^{+}\right] .
$$

Combining this with (6.11), we get

$$
\begin{aligned}
C(0) & =\widetilde{E}\left[\left(X_{0}-q\right)^{+}+\int_{0}^{T} \chi_{[q, \infty)}\left(X_{s}\right) d X_{s}+\sigma^{2} H q^{2} \mathcal{L}_{T}^{(X)}(q)\right] \\
& =\left(X_{0}-q\right)^{+}+\sigma^{2} H q^{2} \widetilde{E}\left[\mathcal{L}_{T}^{(X)}(q)\right]
\end{aligned}
$$

since the expectation of the middle term, a quasi-martingale with respect to $\widetilde{P}$, is zero. 
Following Carr and Jarrow [5], we call $\left(X_{0}-q\right)^{+}$the intrinsic value of the option, and $\sigma^{2} H q^{2} \widetilde{E}^{(H)}\left[\mathcal{L}_{T}^{(X)}(q)\right]$ the time value of the option.

Note that, letting $H \rightarrow 1 / 2$ in Eq.(6.11) we get the price process of a geometric Brownian motion. Taking the expectation of Eq. (6.11), under the risk-free measure for a geometric Brownian motion, the middle term again vanishes, and the expectation of the local time remains in agreement with Lemma A5 of Carr and Jarrow [5]. This case is Proposition 2 of Carr and Jarrow [5]. On the other hand, letting $H \rightarrow 1$ in Eq. (6.11) we get the case of a continuous price process of bounded variation. Then the local time term disappears since $\mathcal{L}_{t}^{(X)}(q)$ is zero for bounded variation processes. This complements the results of Carr and Jarrow [5] and Salopek [22] using Lebesgue and Riemann Stieltjes integrals. In the latter paper, the SLSG portfolio is shown to be self-financing for continuous price process of bounded variation, and so arbitrage opportunities exist for this process.

The put-call parity implies that puts and calls have the same time value. Hence, by the above proposition, we have a value for European put options. Recall that we assume the bond process $A_{t}=1$ for all $t$.

Corollary 6.2 (Put Valuation): Let $P(0)$ be the market price of the European put with maturity date $T$ and exercise price $q$. Assume the fractional Brownian motion model for $H \in(0,1)$. Then

$$
P(0)=\left(q-X_{0}\right)^{+}+\sigma^{2} H q^{2} \widetilde{E}\left[\mathcal{L}_{T}^{(X)}(q)\right]
$$

\section{Appendix}

Proof of Theorem 5.3:

We proceed as in the proof of Theorem 4.1. Let $\left\{f_{n}\right\}$ be a sequence of smooth functions converging to $f$ (as in the proof of Theorem 4.1).

Define $g_{n}(t, y)=f_{n}\left(x \exp \left(\sigma y-\frac{1}{2} \sigma^{2} t^{2 H}+\mu t\right)\right)$. Then by the fractional Itô formula (Lemma 3.1), we have

$$
\begin{aligned}
f_{n}\left(X_{t}\right)=g_{n}\left(t, B_{t}^{(H)}\right)=g_{n}(0,0)+\int_{0}^{t} \frac{\partial g_{n}}{\partial s}\left(s, B_{s}^{(H)}\right) d s \\
+\int_{0}^{t} \frac{\partial g_{n}}{\partial y}\left(s, B_{s}^{(H)}\right) d B_{s}^{(H)}+H \int_{0}^{t} s^{2 H-1} \frac{\partial^{2} g_{n}}{\partial y^{2}}\left(s, B_{s}^{(H)}\right) d s \\
=f_{n}(x)+\int_{0}^{t} f_{n}^{\prime}\left(X_{s}\right) X_{s}\left[-H \sigma^{2} s^{2 H-1} d s+\mu d s+\sigma d B_{s}^{(H)}\right] \\
+H \int_{0}^{t} s^{2 H-1}\left[f_{n}^{\prime \prime}\left(X_{s}\right) X_{s}^{2} \sigma^{2}+f_{n}^{\prime}\left(X_{s}\right) X_{s} \sigma^{2}\right] d s
\end{aligned}
$$




$$
=f_{n}(x)+\int_{0}^{t} f_{n}^{\prime}\left(X_{s}\right) d X_{s}+H \int_{0}^{t} s^{2 H-1} f_{n}^{\prime \prime}\left(X_{s}\right) X_{s}^{2} \sigma^{2} d s .
$$

Proceeding as in the proof of Theorem 4.1,

$$
\begin{aligned}
\int_{0}^{t} f_{n}^{\prime \prime}\left(X_{s}\right) \sigma^{2} X_{s}^{2} H s^{2 H-1} d s & =\int_{0}^{t}\left(\int_{\mathbb{R}} f_{n}^{\prime \prime}\left(X_{s}\right) \sigma^{2} x^{2} \delta\left(X_{s}-x\right) d x\right) H s^{2 H-1} d s \\
& =\sigma^{2} H \int_{\mathbb{R}} f_{n}^{\prime \prime}(x) x^{2} \mathcal{L}_{t}^{(X)}(x) d x \\
& \rightarrow \sigma^{2} H \int_{\mathbb{R}} x^{2} \mathcal{L}_{t}^{(X)}(x) d \nu_{f}(x) \text { as } n \rightarrow \infty .
\end{aligned}
$$

Letting $n \rightarrow \infty$ in (7.1) and using (7.2) we obtain (5.11).

Proof of Corollary 5.4:

In this case $f(x)=|x-z|$ in Theorem 5.3, so $D^{-} f(x)=\operatorname{sign}(x-z)$ and $\nu_{f}(x)=2 \delta_{z}(x)$.

Proof of Corollary 5.5:

In this case $f(x)=(x-z)^{+}$in Theorem 5.3, so $D^{-} f(x)=\chi_{[z, \infty)}$ and $\nu_{f}(x)=\delta_{z}(x)$.

Acknowledgments: We are grateful to Knut Aase, Michel Emery and John van der Hoek for their invaluable discussions.

\section{References}

[1] Alòs, E.; Mazet, O.; Nualart, D. Stochastic calculus with respect to fractional Brownian with Hurst parameter lesser than $\frac{1}{2}$. Stoch. Proc. Appl. 2000, 86, 121-139.

[2] Bender, C. An Itô formula for generalized functionals of a fractional Brownian motion with arbitrary Hurst parameter. Preprint 2002.

[3] Berman, S.M. Local nondeterminism and local times of Gaussian processes. Indiana University Mathematics Journal 1973, 23(1), 69-94.

[4] Biagini, F.; Øksendal, B.; Sulem, A.; Wallner, N. Malliavin calculus for fractional Brownian motion. Preprint 2002.

[5] Carr, P.P.; Jarrow, R.A. The stop-loss-start-gain paradox and option valuation: A new decomposition into intrinsic and time value. The Review of Financial Studies 1990, 3, 469-492.

[6] Coutin, L.; Nualart, D.; Tudor, C.A. Tanaka formula for the fractional motion. Stochastic Processes and their Applications 2001, 94(2), 301-315. 
[7] Dai, W.; Heyde, C.C. Itô's formula with respect to fractional Brownian motion and its applications. Journal of Appl. Math. and Stoch. An. 1996 9, 439-448.

[8] Duncan, T. E.; Hu, Y.; Pasik-Duncan, B. Stochastic calculus for fractional Brownian motion. I. Theory. SIAM J. Control Optim. 2000, 38, 582-612.

[9] Elliott, R.J.; van der Hoek, J. Fractional Brownian motion and financial modeling. In Trends in Mathematics. Mathematical Finance, Workshop of the Mathematical Finance Research Project, Konstanz, Germany, October 5-7, 2000, M. Kohlmann and S. Tang, Eds; Birkhäuser Verlag Basel, Switzerland, 2001, 140-151.

[10] Elliott, R.J.; van der Hoek, J. A generalized fractional white noise theory and applications to finance. Preprint 2002.

[11] Hu, Y. Self-Intersection Local Time of Fractional Brownian Motions - Via Chaos Expansion. J. Math. Kyoto Univ. 2001 41(2), 233-250.

[12] Holden, H.; Øksendal, B.; Ubøe, J.; Zhang, T. Stochastic Partial Differential Equations. Birkhäuser, Boston 1996.

[13] Hu, Y.; Øksendal, B. Fractional white noise calculus and application to finance. Preprint, University of Oslo, 10/1999.

[14] Hu, Y.; Øksendal, B. Chaos expansion of local time of fractional Brownian motions. Stochastic Analysis and Applications 2002, 20(4), 815-837.

[15] Hu, Y.; Øksendal, B.; Sulem, A. Optimal portfolio in a fractional Black \& Scholes market. In Mathematical Physics and Stochastic Analysis: Essays in Honor of Ludwig Streit; S. Albeverio et al Eds.; World Scientific, River Edge, N.J. 2000, 267-279.

[16] Hu, Y.; Øksendal, B.; Sulem, A. Optimal assumption and portfolio in a Black-Scholes market driven by fractional Brownian motion. Preprint, University of Oslo 23/2000.

[17] Hu, Y.; Øksendal, B.; Sulem, A. A stochastic maximum principle for processes driven by fractional Brownian motion. Preprint, University of Oslo 24/2000.

[18] Lin, S.J. Stochastic analysis of fractional Brownian motion, fractional noises and applications. SIAM Review 1995, 10, 422-437.

[19] Mandelbrot, B.B.; van Ness, J.W. Fractional Brownian motion, fractional noises and applications. SIAM Review 1968, 10, 422-437.

[20] Norvaiša, R. p-variation and integration of sample functions of stochastic processes. In Probability Theory and Mathematical Statistics. Proc. of the Seventh Vilnius Conference (1998), B. Grigelionis et al Eds.; TEV Vilnius Lithuania, 1999, 521-540.

[21] Revuz, D.; Yor, M. Continuous Martingales and Brownian Motions, Third edition, Springer, Berlin NY 1999. 
[22] Salopek, D.M. A new class of nearly self-financing strategies. Statistics 6 Probability Letters 2002, 56, 69-75.

[23] van der Hoek, J. Private communication 2002.

Yaozhong $\mathrm{Hu}$

Department of Mathematics

University of Kansas

405 Snow Hall

Lawrence, Kansas 66045-2142, USA.

Email: hu@math.ukans.edu

Bernt Øksendal

Department of Mathematics Norwegian School of Economics

University of Oslo

Box 1053 Blindern and Business Administration

N-0316 Oslo, Norway

Helleveien 30

N-5045 Bergen, Norway

Email: oksendal@math.uio.no

Donna Mary Salopek

School of Mathematics

University of New South Wales

Sydney, NSW 2052

Australia

Email: donnas@maths.unsw.edu.au 